\title{
Proposition of a Stepwise Diagnosis Method for a Cracked Beam using a Force Identification Approach*
}

\author{
Shozo KAWAMURA**, Kazuma SAKAI**, Yuto SUZUKI** \\ and Hirofumi MINAMOTO** \\ **Department of Mechanical Engineering, Toyohashi University of Technology, \\ 1-1 Tempaku, Toyohashi, Aichi, 441-8580, Japan \\ E-mail: kawamura@me.tut.ac.jp
}

\begin{abstract}
Machine condition monitoring and diagnosis have become increasingly important, and the application of these processes to stationary structures and rotating machinery has been widely investigated. The authors previously proposed a stepwise diagnosis method. In that method, the location of the abnormality is first estimated using the force identification approach, and then the cause and the severity of the abnormality are identified. But the method was applicable for an abnormality of linear property. In this study, a stepwise diagnostic approach for a beam with an abnormality of nonlinear property, that is, a breathing crack, is proposed. In this method, the location and the severity of abnormality are identified by using the multi-frequencies of response under the abnormal condition. Using the numerical example of a uniform beam fixed at both ends, we confirmed the validity of the method and determined that the selection of the excitation frequency was very important. Moreover, we showed the applicability of the method by using experimental data. We conclude that the proposed diagnosis method is feasible for identifying the abnormality of a breathing crack.
\end{abstract}

Key words: Model-Based Diagnosis Approach, Inverse Analysis, Force Identification, Modal Analysis, Beam, Breathing Crack

\section{Introduction}

Machine condition monitoring and diagnosis have become increasingly important, and the application of these processes to beam structures and rotating machinery has been widely investigated. At the early stage of diagnosis, abnormal data is encountered, and a primary diagnosis is required to identify the location, the cause and the severity of the abnormality.

There have been many studies about the primary diagnosis of abnormality. Some of them use a knowledge-based approach, i.e., an expert system in which fault-symptom matrices, if-then rules, fuzzy logic or neural networks are used ${ }^{(1)-(5)}$. Others use a model-based approach in which the abnormal response is calculated from a mathematical model having a certain cause of abnormality with its adequate severity, and the residual between the measured and calculated response is checked; the correct cause and the severity of abnormality can then be identified as the cause and the severity leading to the minimum residual. Many studies use a model-based approach to the crack diagnosis of a beam ${ }^{(6)-(15)}$ and to the crack or unbalance diagnosis of a rotor system ${ }^{(16)-(19)}$. The authors previously proposed a stepwise primary diagnostic method ${ }^{(20)}$ that was a model-based approach. At the first step of the method, the location of abnormality is estimated using the force 
identification approach. At the second step, the cause and the severity of abnormality are identified using the mathematical model having a certain cause and severity of abnormality. The distinguishing feature at the estimation process of the location of abnormality is that the abnormality is considered as a virtual additional external force in the early stage of abnormality. A numerical example showed that the location, the cause and the severity of abnormality could be identified with sufficient accuracy, and that this method was useful for primary diagnosis. This was the original study of our stepwise diagnosis method. The abnormality considered in the study was the one with a linear property, i.e., an additional mass or a change of rigidity, and the method was applied to a simple structure like a uniform beam.

As a next step, we developed a new diagnostic approach to increase the robustness of our stepwise diagnosis method, where the mathematical model was modified based on the difference between the measured response and the calculated one ${ }^{(21)}$. The validity and applicability of the proposed method were shown for the experimental data of a free-free uniform beam. The authors also proposed a new regularization method in the force identification procedure, and the method was proved to be feasible for actual application ${ }^{(22)}$. As another application of the diagnosis method, we considered a rotor system ${ }^{(23)}$. Experiments showed that the method was valid in the abnormal cases of unbalance and stationary rubbing.

In this study, we propose a stepwise diagnostic approach specifically for a beam with an abnormality of nonlinear property. As an example of abnormality, a breathing crack is considered in this study, but the method is applicable for other causes of abnormality. The forced response of a beam under the normal condition is synchronous with the external force, but in the case of a beam with a breathing crack, the characteristic frequency components of the forced response can be observed under the specific frequency of the external force. We propose a new estimation method of the location of abnormality from the multi-frequencies of response under the abnormal condition using a force identification approach.

We used a numerical example to check the validity of our method. A uniform beam fixed at both ends was considered, and a breathing crack was modelled as an equivalent spring ${ }^{(24)}$. The forced response was calculated by using the finite element method (FEM) based on the mixed variational principle ${ }^{(25)}$. After that, the applicability of the method was investigated using the experimental data.

In this study, we considered the breathing crack as an abnormality, while our method isn't limited to the one. We are able to consider the various causes of abnormality at the diagnosis process.

\section{Proposition of a fault diagnosis method}

In this section, we describe how the stepwise diagnosis method ${ }^{(20)}$ is improved for a beam with a breathing crack.

\subsection{Construction of the mathematical model}

In previous studies ${ }^{(20)-(23)}$, the structure to be diagnosed was modelled as having no damping, because the response data far from the resonance frequency, $\omega_{r}$, was almost the same in the cases with and without damping for a structure with slight damping.

In the case of a breathing crack, the secondary resonance will occur near the half of the resonance frequency. In our diagnosis procedure, the frequency of the external force, $\omega$, is set near the half of $\omega_{r}$, and the secondary resonance will be utilized. The frequency of the secondary resonance is near $\omega_{r}$. Thus, the virtual additional external force of frequency, $2 \omega \approx \omega_{r}$, has to be identified, so that the structure is modelled with damping.

In this diagnosis system, a vibration test will be performed at regular intervals to 
monitor the health of the structure. The external force for the vibration test can be measured, and the responses are measured at several points. The mass matrix, $[M]$, the damping matrix, $[D]$, and the stiffness matrix, $[K]$, of the structure under the normal condition have been constructed in advance by using the FEM, and they are modified by the model update procedure so that the calculated response agrees with the experimental one.

A harmonic excitation with frequency $\omega$ is considered as the external force. The equation of motion and the output equation under the normal condition are obtained as follows:

$$
\left([K]-\omega^{2}[M]+i \omega[D]\right)\left\{X_{n}\right\}=\{F\}, \quad\left\{X_{m}\right\}=[C]\left\{X_{n}\right\},
$$

where $\left\{X_{n}\right\}$ and $\{F\}$ are the magnitude of response and external force, respectively. $\left\{X_{m}\right\}$ is the magnitude of the measured response, and $[C]$ is the coefficient matrix that expresses the measurement positions.

\subsection{Estimation of the location of abnormality}

In the abnormal condition, the response changes because the dynamic characteristics change due to abnormality, even if the same external force acts on the structure in the vibration test. And if the abnormality has a nonlinear property, the response under the abnormal condition may have various frequency components. For example, in the case of a breathing crack on the beam, because the rigidity at the crack location changes twice during one period, not only the synchronous component of the external force, $\omega$, but also the second-order component $2 \omega$ can be observed. When the excitation frequency is near the half of the resonance frequency of the beam, the $2 \omega$ component is significantly excited, and that is the secondary resonance.

In this study, the change of response is considered to be the result of a virtual additional external force exerted on the normal structure. Under the normal condition, the frequency component $\omega$ of the response can be observed for the harmonic excitation with frequency $\omega$, while under the abnormal condition of a breathing crack, the frequency components $\omega$ and $2 \omega$ in the response can be measured for the same excitation condition. Therefore, the next equations are obtained:

$$
\begin{aligned}
& \left([K]-\omega^{2}[M]+i \omega[D]\right)\left\{X_{\omega, n}\right\}=\{F\}+\left\{F_{\omega}\right\}, \\
& \left([K]-(2 \omega)^{2}[M]+i(2 \omega)[D]\right)\left\{\Delta X_{2 \omega, n}\right\}=\left\{F_{2 \omega}\right\}
\end{aligned}
$$

where

$$
\begin{aligned}
& \left\{X_{\omega, n}\right\}=\left\{X_{n}\right\}+\left\{\Delta X_{\omega, n}\right\}, \quad\left\{\Delta X_{\omega, m}\right\}=[C]\left\{\Delta X_{\omega, n}\right\}, \\
& \left\{\Delta X_{2 \omega, m}\right\}=[C]\left\{\Delta X_{2 \omega, n}\right\} .
\end{aligned}
$$

Here there is no component $2 \omega$ under the normal condition; the second-order component is the response change $\left\{\Delta X_{2 \omega, n}\right\}$ in Eq. (2). Moreover, an abnormality in the early stage occurs locally in the structure, so the additional external force is considered to act only on the $i$-th element of the FEM. The force elements can be identified using the relationship

$$
\left\{\Delta X_{\omega, m}\right\}=[H(\omega)]\left\{F_{\omega, i}\right\}, \quad\left\{\Delta X_{2 \omega, m}\right\}=[H(2 \omega)]\left\{F_{2 \omega, i}\right\}
$$

where $[H(\omega)]$ and $[H(2 \omega)]$ are compliance matrices between the measured responses and the assumed external force. $\left\{F_{\omega, i}\right\}$ and $\left\{F_{2 \omega, i}\right\}$ are the additional external force to be identified. The singular value decomposition is applied to solve Eq.(4). Even if some singular values are significantly small, in the ideal condition without measurement noise and modelling error, the exact response changes can be obtained. In the actual application, however, because measurement noise or modelling error cannot be avoided, the truncation of small singular values is needed when the compliance matrices $[H(\omega)]$ and $[H(2 \omega)]$ are often ill-posed condition. The additional external forces $\left\{\widetilde{F}_{\omega, i}\right\}$ and $\left\{\widetilde{F}_{2 \omega, i}\right\}$ are identified by solving Eq.(4) with the truncation of small singular values, and $\left\{\Delta X_{\omega, i, m}\right\}$ and $\left\{\Delta X_{2 \omega, i, m}\right\}$ can be obtained by $[H(\omega)]\left\{\widetilde{F}_{\omega, i}\right\}$ and $[H(2 \omega)]\left\{\widetilde{F}_{2 \omega, i}\right\}$, respectively 
The next objective functions, $J_{\omega, 1}$ and $J_{2 \omega, 1}$, are calculated for every element number $i$, as follows:

$$
J_{\omega, 1}=\left|\left\{\Delta X_{\omega, m}\right\}-\left\{\Delta X_{\omega, i, m}\right\}\right|, \quad J_{2 \omega, 1}=\left|\left\{\Delta X_{2 \omega, m}\right\}-\left\{\Delta X_{2 \omega, i, m}\right\}\right|
$$

where $|\bullet|$ means the Euclid norm, and it is considered that the abnormality will occur at the element where $J_{\omega, 1}$ and $J_{2 \omega, 1}$ are significantly small.

When the ideal mathematical model is constructed and the response data without noise can be used, the element number where the objective functions are small is expected to be the same for $J_{\omega, 1}$ and $J_{2 \omega, 1}$, but in the actual application, the element number may be different, and thus the final location of the abnormality must be determined by synthesizing two results from $J_{\omega, 1}$ and $J_{2 \omega, 1}$.

\subsection{Identification of the cause and the severity of abnormality}

After the location of abnormality is estimated as the $I$-th element, we consider the possible causes of the abnormality. One cause is set as $(j)$. A mathematical model with the cause of abnormality $(j)$ having a certain severity in the $I$-th element is constructed.

In this study, because the nonlinear phenomenon, which is a $2 \omega$ component, is observed under the abnormal condition, the possible cause of abnormality may have a nonlinear property. In this case we can obtain the synchronous and the higher-order components with the excitation frequency from the calculated response. The synchronous and the second-order components with the excitation frequency are set as $\left\{X_{\omega, I, n}^{(j)}\right\}$ and $\left\{\Delta X_{2 \omega, I, n}^{(j)}\right\}$, respectively. Then, the response changes of the frequency component $\omega$ and $2 \omega$ at the measurement points are obtained as follows:

$$
\left\{\Delta X_{\omega, I, m}^{(j)}\right\}=[C]\left(\left\{X_{\omega, I, n}^{(j)}\right\}-\left\{X_{n}\right\}\right),\left\{\Delta X_{2 \omega, I, m}^{(j)}\right\}=[C]\left\{\Delta X_{2 \omega, I, n}^{(j)}\right\} .
$$

The nonlinear forced responses are obtained by a usual calculation method, i.e., the method of multiple scales, the method of harmonic balance and so on.

The next objective functions, $J_{\omega, 2}$ and $J_{2 \omega, 2}$, are calculated as follows:

$$
J_{\omega, 2}=\left|\left\{\Delta X_{\omega, m}\right\}-\left\{\Delta X_{\omega, I, m}^{(j)}\right\}\right|, J_{2 \omega, 2}=\left|\left\{\Delta X_{2 \omega, m}\right\}-\left\{\Delta X_{2 \omega, I, m}^{(j)}\right\}\right|,
$$

and the cause and the severity of abnormality are identified when $J_{\omega, 2}$ and $J_{2 \omega, 2}$ are significantly small. In the case of an actual application, the final results, which are the cause and the severity of abnormality, must be determined by synthesizing two results from $J_{\omega, 2}$ and $J_{2 \omega, 2}$.

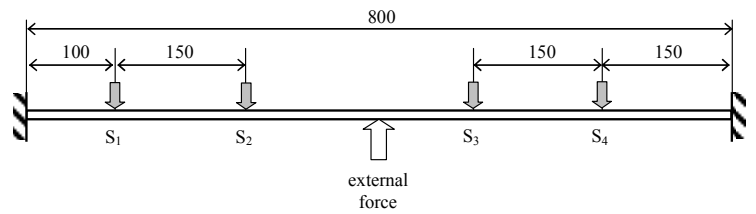

Fig. 1 A uniform beam fixed at both ends

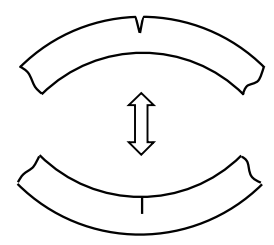

Fig. 2 Opening and closing of a breathing crack in a vibrating beam

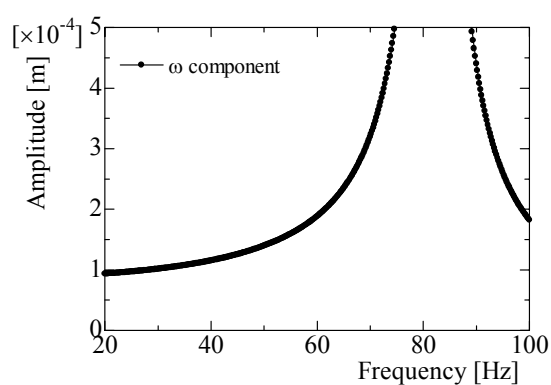

Fig. 3 Resonance curve at the third sensor, $\mathrm{S}_{3}$, in the normal condition 


\section{Validity of the proposed method}

We first checked the validity of the proposed method by using a numerical example.

\subsection{A beam structure to be diagnosed}

Figure 1 shows the model to be diagnosed, which consists of a uniform beam fixed at both ends whose length, $l$, is $800.0 \mathrm{~mm}$, width, $w$, is $15.0 \mathrm{~mm}$, and thickness, $t$, is 10.0 $\mathrm{mm}$.

To construct a mathematical model, we divide the beam into 80 elements and use the FEM to obtain the characteristic matrices using a beam element. The proportional damping

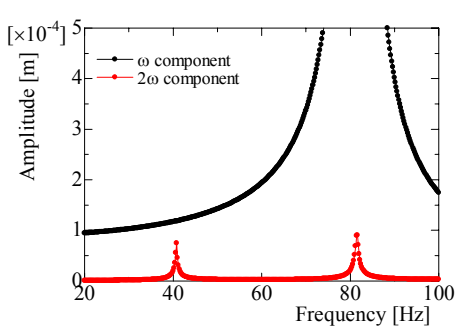

(a) The crack is located at $300 \mathrm{~mm}$ (No. 30 node).

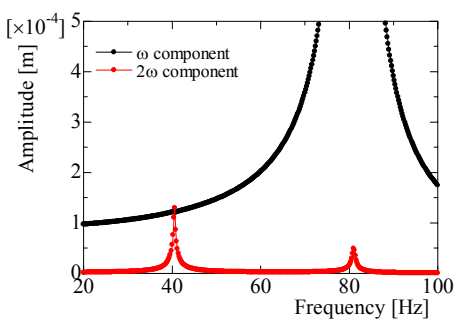

(c) The crack is located at $400 \mathrm{~mm}$ (No. 40 node).

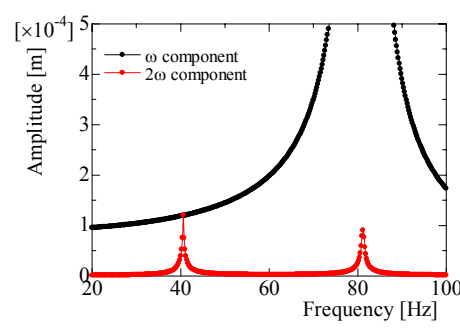

(b) The crack is located at $350 \mathrm{~mm}$ (No. 35 node).

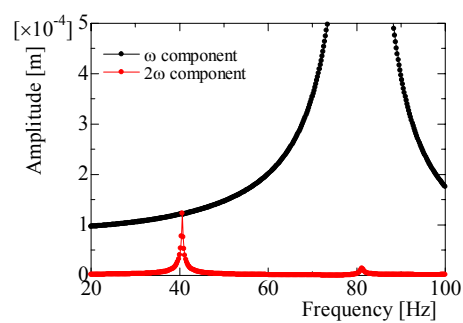

(d) The crack is located at $450 \mathrm{~mm}$ (No. 45 node).

Fig.4 Resonance curve at the third sensor, $\mathrm{S}_{3}$, in the case of the ratio of the crack depth $\alpha=0.5$

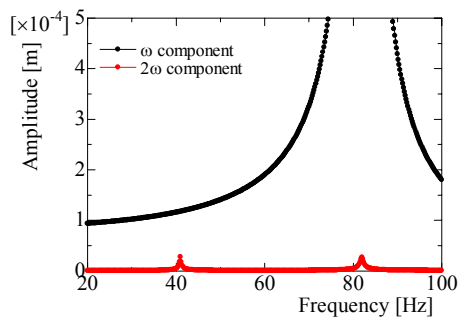

(a) The crack is located at $300 \mathrm{~mm}$ (No. 30 node).

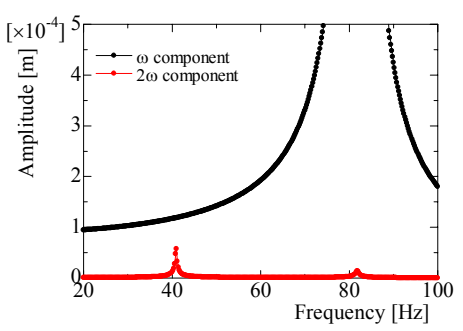

(c) The crack is located at $400 \mathrm{~mm}$ (No. 40 node).

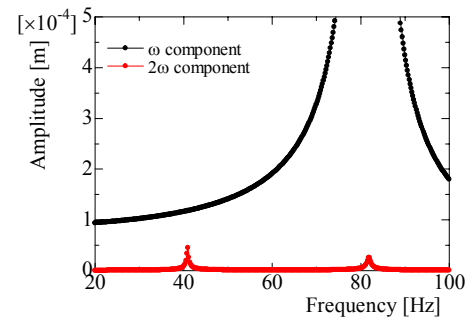

(b) The crack is located at $350 \mathrm{~mm}$ (No. 35 node).

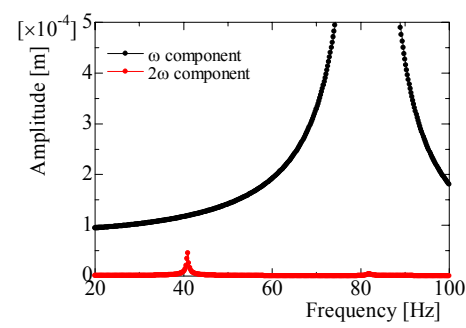

(d) The crack is located at $450 \mathrm{~mm}$ (No. 45 node).

Fig. 5 Resonance curve at the third sensor, $\mathrm{S}_{3}$,

in the case of the ratio of the crack depth $\alpha=0.3$ 
matrix $[D]=\beta[K]$ is adopted. The density, $\rho$, the Young's modulus, $E$, and Poisson's ratio, $v$, of the beam are $7.83 \times 10^{3} \mathrm{~kg} / \mathrm{m}^{3}, 205 \mathrm{GPa}$, and 0.3 , respectively. The coefficient of damping, $\beta$, is set as $1.6 \times 10^{-5} \mathrm{sec}$. The lower two natural frequencies are $82.18 \mathrm{~Hz}$ and $226.54 \mathrm{~Hz}$.

The external force for the vibration test acts at the center of the beam, whose amplitude

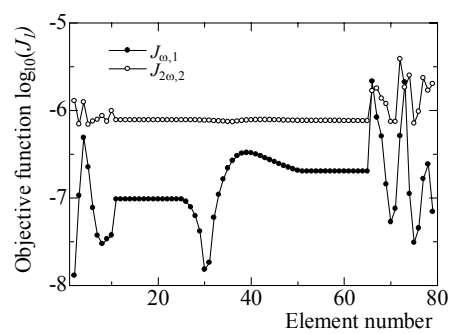

(a) The crack : at $300 \mathrm{~mm}$ (No. 30 node), the excitation frequency : $40.8 \mathrm{~Hz}$.

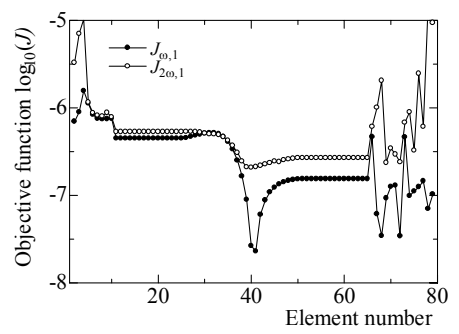

(c) The crack: at $400 \mathrm{~mm}$ (No. 40 node), the excitation frequency : $40.6 \mathrm{~Hz}$.

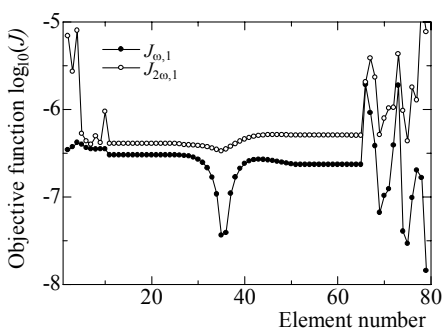

(b) The crack : at $350 \mathrm{~mm}$ (No. 35 node), the excitation frequency : $40.6 \mathrm{~Hz}$.

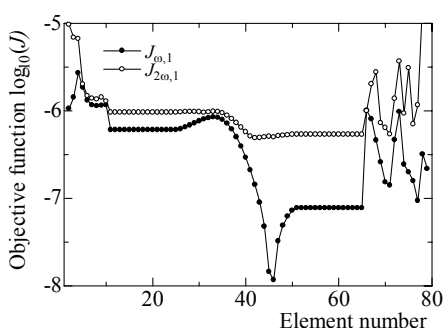

(d) The crack : at $450 \mathrm{~mm}$ (No. 45 node), the excitation frequency : $40.6 \mathrm{~Hz}$.

Fig.6 Estimation of the location of abnormality at the excitation frequency where the $2 \omega$ component is most excited in the case of the ratio of the crack depth $\alpha=0.5$.

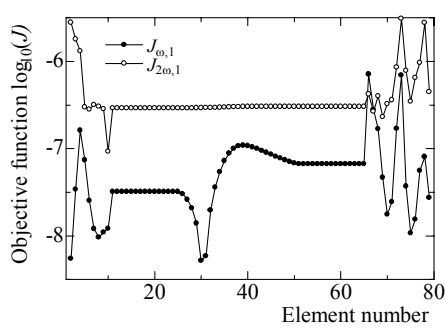

(a) The crack : at $300 \mathrm{~mm}$ (No. 30 node), the excitation frequency : $41.0 \mathrm{~Hz}$.

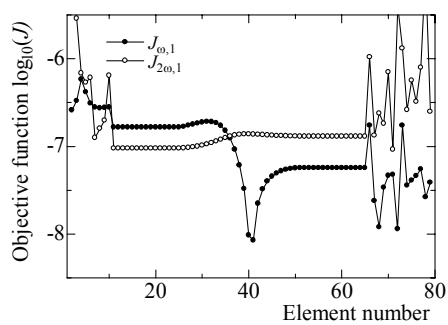

(c) The crack: at $400 \mathrm{~mm}$ (No. 40 node), the excitation frequency : $41.0 \mathrm{~Hz}$.

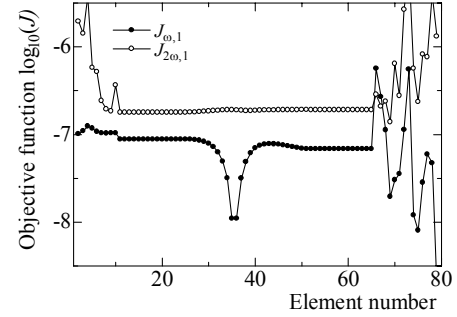

(b) The crack : at $350 \mathrm{~mm}$ (No. 35 node), the excitation frequency : $41.0 \mathrm{~Hz}$.

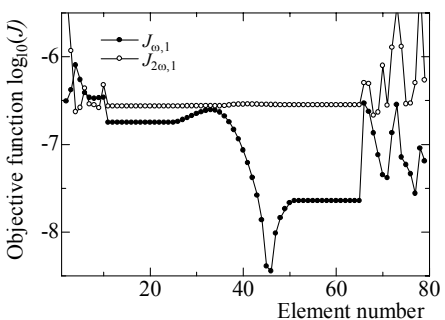

(d) The crack : at $450 \mathrm{~mm}$ (No. 45 node), the excitation frequency: $41.0 \mathrm{~Hz}$.

Fig.7 Estimation of the location of abnormality at the excitation frequency where the $2 \omega$ component is most excited in the case of the ratio of the crack depth $\alpha=0.3$. 
is $10.0 \mathrm{~N}$. The responses are measured at four points, which are $100 \mathrm{~mm}, 250 \mathrm{~mm}, 500 \mathrm{~mm}$, and $650 \mathrm{~mm}$ from the left end.

\subsection{Modelling of the breathing crack}

In this study, we considered a breathing crack as an abnormality, the modelling of which we explain here. When a beam with a breathing crack vibrates, the crack opens and closes repeatedly. The opening and closing of the crack depends on the sign of the bending moment at the crack location. When the crack opens, the flexural rigidity at the crack

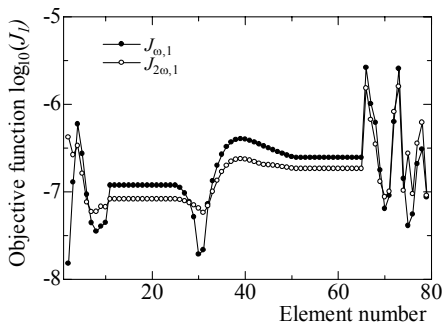

(a) The crack : at $300 \mathrm{~mm}$ (No. 30 node), the excitation frequency : $40.0 \mathrm{~Hz}$.

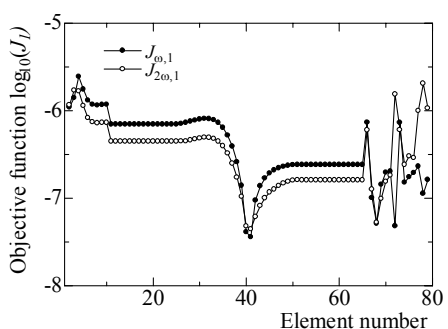

(c) The crack : at $400 \mathrm{~mm}($ No. 40 node), the excitation frequency: $40.0 \mathrm{~Hz}$.

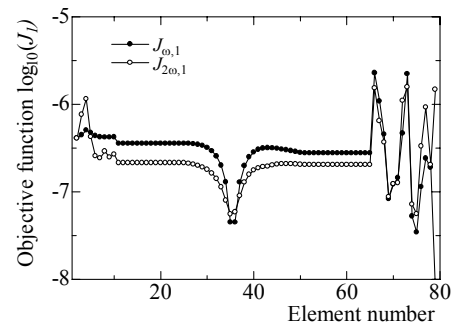

(b) The crack : at $350 \mathrm{~mm}$ (No. 35 node), the excitation frequency : $40.0 \mathrm{~Hz}$.

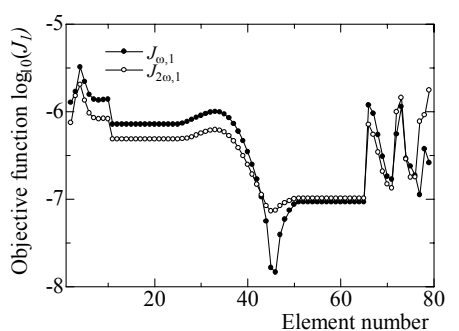

(d) The crack : at $450 \mathrm{~mm}($ No. 45 node), the excitation frequency: $40.0 \mathrm{~Hz}$.

Fig.8 Estimation of the location of abnormality at the excitation frequency $40.0 \mathrm{~Hz}$ in the case of the ratio of the crack depth $\alpha=0.5$.

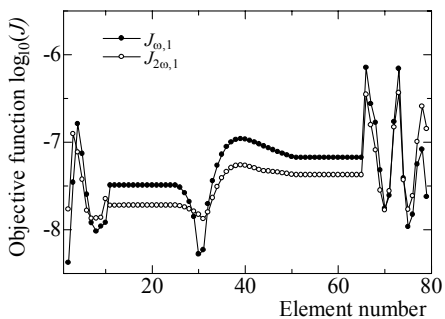

(a) The crack : at $300 \mathrm{~mm}$ (No. 30 node), the excitation frequency: $40.0 \mathrm{~Hz}$.

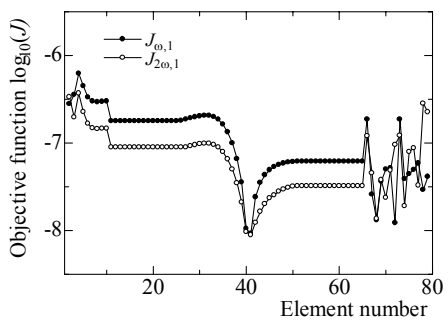

(c) The crack : at $400 \mathrm{~mm}($ No. 40 node), the excitation frequency : $40.0 \mathrm{~Hz}$.

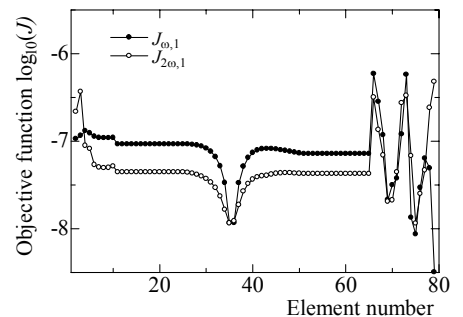

(b) The crack : at $350 \mathrm{~mm}$ (No. 35 node), the excitation frequency : $40.0 \mathrm{~Hz}$.

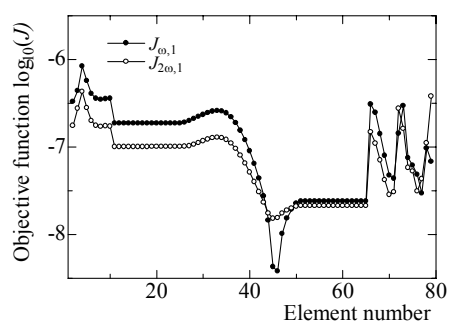

(d) The crack : at $450 \mathrm{~mm}$ (No. 45 node), the excitation frequency : $40.0 \mathrm{~Hz}$.

Fig.9 Estimation of the location of abnormality at the excitation frequency $40.0 \mathrm{~Hz}$ in the case of the ratio of the crack depth $\alpha=0.3$. 
location decreases locally, so we model the decrease in the flexural rigidity by an equivalent spring, as shown in Fig. ${ }^{(24)}$. The equivalent spring constant, $k_{c}$, is expressed as follows:

$$
\frac{1}{k_{c}}=\frac{6 \pi\left(1-v^{2}\right) t}{E I} \phi(\alpha)
$$

where $v$ is the Poisson ratio, EI is the flexural rigidity, $\alpha$ is the ratio of the crack depth to the beam thickness, and the function $\phi(\alpha)$ is expressed as follows:

$$
\begin{aligned}
\phi(\alpha) & =0.6272 \alpha^{2}-1.04533 \alpha^{3}+4.5948 \alpha^{4}-9.9736 \alpha^{5}+20.2948 \alpha^{6} \\
& -33.0351 \alpha^{7}+47.1063 \alpha^{8}-40.7556 \alpha^{9}+19.6 \alpha^{10}
\end{aligned} .
$$

We assume that the flexural rigidity of the beam is the same as that of the beam without a crack when the crack is closed. Moreover, we assume that the crack does not affect the mass of the beam.

\subsection{Normal and abnormal responses}

The steady-state response of a beam with a breathing crack was calculated by using the FEM based on the mixed variational principle. The method was developed by K. Kamiya and T. Yoshinaga ${ }^{(25)}$. As an alternative method, the dynamic response was calculated by a numerical integration of the differential equation, and we confirmed the agreement of the results obtained by these two methods.

In the normal case, the amplitude of the steady-state response is as shown in Fig. 3 when the magnitude of the external force is $10.0 \mathrm{~N}$. The results of a beam with a breathing crack with a ratio of the depth $\alpha=0.5$ is shown in Fig. 4, and that for a ratio of the depth $\alpha=0.3$ is shown in Fig. 5 . The crack is located at $300 \mathrm{~mm}, 350 \mathrm{~mm}, 400 \mathrm{~mm}$, or $450 \mathrm{~mm}$ from the left end, and the corresponding node numbers are $30,35,40$, and 45 , respectively. For all cases, when the excitation frequency is near $40.0 \mathrm{~Hz}$, which is a half of the resonance frequency, the second-order harmonics is significantly excited, which is the secondary resonance. In this study, we are actively utilizing this phenomenon.

\subsection{Estimation of the location of abnormality}

The location of abnormality was estimated by the diagnostic method described in Sec. 2.2 using the response data without noise. In the inverse analysis of Eq. (4), three singular values in $[H(\omega)]$ were adopted, because the crack location was set between the second and the third sensor. In our previous study ${ }^{(22)}$, we determined that the optimum number of the singular value depends on the sensor location and the location of abnormality.

First, we identified the location of abnormality at the excitation frequency where the $2 \omega$ component was most excited. The result in the case of $\alpha=0.5$ is shown in Fig. 6, and the case of $\alpha=0.3$ is shown in Fig. 7. The horizontal axis denotes the element number in which the virtual additional external force acts, and the vertical axis shows the objective function of Eq. (5). As shown in Fig. 6(a), for example, the excitation frequency is $40.8 \mathrm{~Hz}$ from Fig. 4(a), and the objective function $J_{\omega, 1}$ is significantly small at the No. 30 and No. 31 elements. The crack is located $300 \mathrm{~mm}$ from the left end, which is the 30-th nodal point, between the No. 30 and No. 31 elements. Thus the result of $J_{\omega, 1}$ indicates that the exact

Table 1 The estimated location of abnormality

\begin{tabular}{ccc}
\hline $\begin{array}{c}\text { Exact location of crack } \\
\text { (node number) }\end{array}$ & $\begin{array}{c}\alpha=0.5 \\
\text { (node number) }\end{array}$ & $\begin{array}{c}\alpha=0.3 \\
\text { (node number) }\end{array}$ \\
\hline $300 \mathrm{~mm}$ (No. 30) & 30 or 31 (No. 30) & 30 or 31 (No. 30) \\
\hline $350 \mathrm{~mm}$ (No. 35) & 35 or 36 (No. 35) & 35 or 36 (No. 35) \\
\hline $400 \mathrm{~mm}($ No. 40) & 40 or 41 (No. 40) & 40 or 41 (No. 40) \\
\hline $450 \mathrm{~mm}($ No. 45$)$ & 45 or 46 (No. 45) & 45 or 46 (No. 45) \\
\hline
\end{tabular}


location of abnormality can be obtained. In Fig. 6(a), the objective function $J_{2 \omega, 1}$ does not show the minimum value. This is because the frequency $2 \omega$ is very close to the resonance frequency where the vibration shape is similar to the first vibration mode, and thus, wherever the additional force acts on the beam, the vibration shape does not change. This result indicates that the frequency where the $2 \omega$ component is most excited is not always appropriate for the excitation frequency for diagnosis. The relationship of the excitation frequency and the accuracy of diagnosis is important for an actual application. The quantitative consideration will be a future study.

Next, we identified the location of abnormality at $40.0 \mathrm{~Hz}$, and the results are shown in Figs. 8 and 9. These figures show that the location of abnormality could be estimated for all cases, and the final results as the smallest objective function are shown in Table 1. This result shows that the excitation frequency for a vibration test has to be carefully selected for actual application. There is no quantitative instruction for selecting the excitation frequency, so that trial and error will be necessary for actual application.

The next step is the identification of the cause and the severity of abnormality. At the step, the mathematical model having a possible cause of abnormality with its adequate severity is constructed, and then the response is calculated using a numerical method. In the numerical example using the ideal data without noise, it is clear that the exact cause and severity of abnormality can be easily obtained when a crack is assumed as a cause of abnormality. Therefore the next step is to check the validity of the identification of abnormality using experimental data.

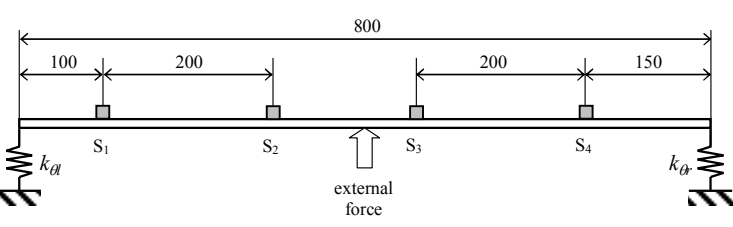

(a) Overview of the experimental setup

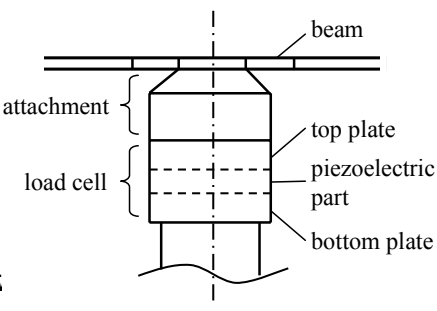

(b) Detailed view of the joint part

Fig.10 Experimental setup

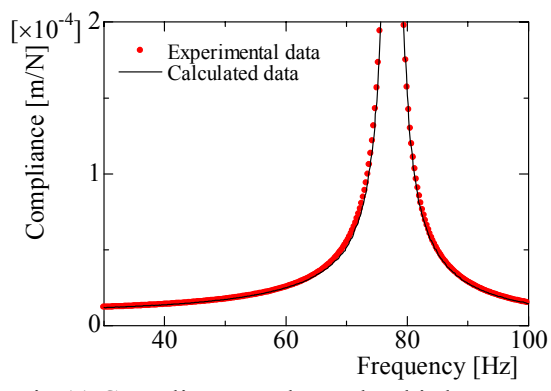

Fig. 11 Compliance at the at the third sensor, $\mathrm{S}_{3}$, in the normal condition

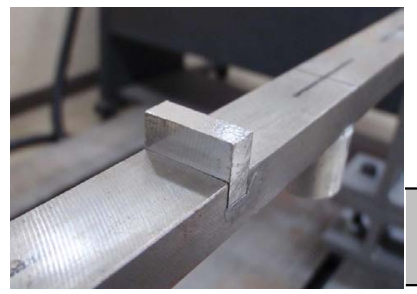

(a) Making a breathing crack

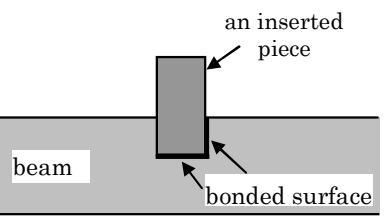

(b) The detail of the crack part

Fig.12 Crack part 


\section{Applicability of the proposed method}

To check the applicability of the proposed method, we diagnose the actual beam with a breathing crack.

\subsection{Experimental setup and its mathematical model}

A uniform steel beam (SS400) fixed at both ends is used as shown in Fig. 10(a). An exciter (IMV m030/MA1) is connected with the beam as shown in Fig.10(b) at the center of the beam. A load cell (PCB 20801) is attached between the exciter and an attachment. The beam is $800.0 \mathrm{~mm}$ long, $15.0 \mathrm{~mm}$ wide, and $10.0 \mathrm{~mm}$ thick. The properties are the same as the numerical example, but the coefficient of damping is set as $7.8 \times 10^{-6} \mathrm{sec}$. Four accelerometers (PCB 353B15) are attached to the beam at $100 \mathrm{~mm}, 300 \mathrm{~mm}, 450 \mathrm{~mm}$, and $650 \mathrm{~mm}$ from the left end.

The mathematical model in the normal condition was constructed using the FEM. The beam is divided into 160 elements, and the masses of the attachment, $18.31 \mathrm{~g}$, and the accelerometers, $2.48 \mathrm{~g}$, were considered in the mathematical model. Both ends were fixed in the translation and supported elastically in the rotation. The properties of the rotational springs were determined to agree with the experimental data, $k_{\theta l}=k_{\theta r}=5.125 \times 10^{4}$ $\mathrm{Nm} / \mathrm{rad}$. The frequency response function at $450 \mathrm{~mm}$ from the left end is shown in Fig. 11. As shown in this figure, the resonance frequency was $77.5 \mathrm{~Hz}$ in the normal condition.

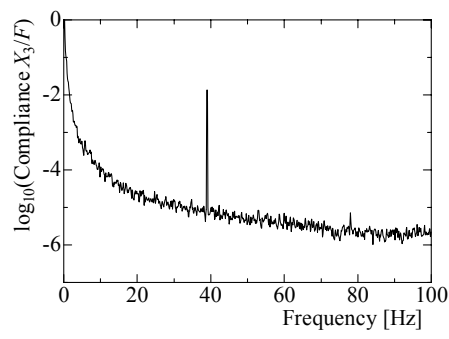

(a) Normal condition

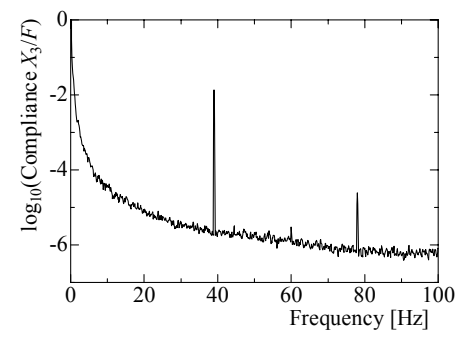

(b) Abnormal condition

Fig. 13 The compliance at the third sensor, $\mathrm{S}_{3}$, at the excitation frequency $39.0 \mathrm{~Hz}$

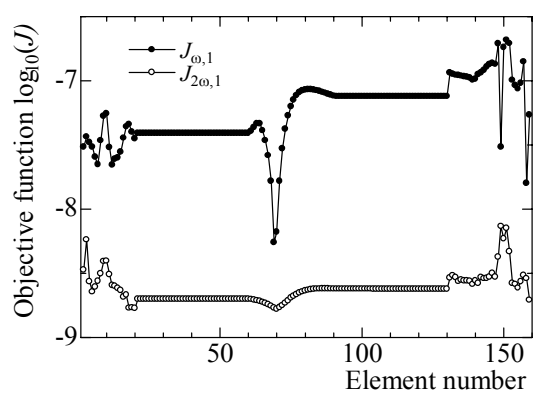

Fig.14 The estimation of the location of abnormality

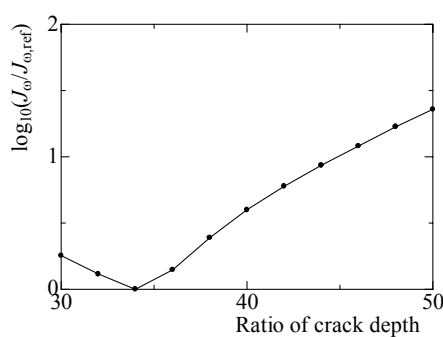

(a) Objective function using the $\omega$ component

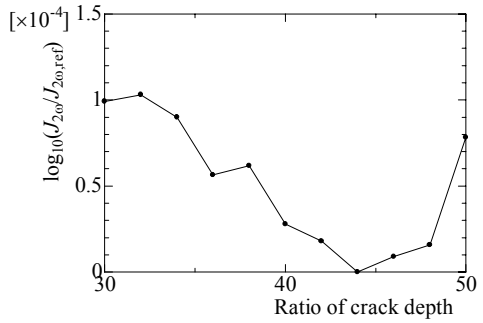

(b) Objective function using the $2 \omega$ component

Fig. 15 The identification of the severity of the crack 
Next we explain the breathing crack. The depth of the crack is $40 \%$ of the beam thickness, and it is located $350 \mathrm{~mm}$ from the left end, between the 70th and 71st elements. The beam was cut to a depth of $4.0 \mathrm{~mm}$ at the $71 \mathrm{st}$ element, and a steel piece of $5 \times 10 \times 15$ $\mathrm{mm}$ was put into the groove, as shown in Fig. 12(a). Two sides of the groove were bonded with the piece, while one side was free, as shown in Fig. 12(b). The piece is $6 \mathrm{~mm}$ larger than the depth of the groove, because the piece and the groove are perfectly connected over the groove. Therefore, in the normal condition, an additional mass was considered at the 71 st element in the mathematical model and the experimental setup.

\subsection{Estimation of the location and severity of abnormality}

From the vibration test, we observed the second harmonics of the external force around $40.0 \mathrm{~Hz}$. We set the excitation frequency as $39.0 \mathrm{~Hz}\left(=\omega_{0}\right)$, and the response is shown in Fig. 13. In the normal condition, the magnitude of the external force at $\omega_{0}$ was $12.6 \mathrm{~N}$, so the magnitude of the response was divided by $12.6 \mathrm{~N}$ and the $2 \omega_{0}$ component was also divided by $12.6 \mathrm{~N}$. In the abnormal condition, the magnitude of the external force at $\omega_{0}$ was $40.5 \mathrm{~N}$, so the components $\omega_{0}$ and $2 \omega_{0}$ were divided by $40.5 \mathrm{~N}$. The response change was determined by the compliance data so that the difference of the magnitude of the external force for the normal and the abnormal condition had no effect on the identification of the location of abnormality. Using these data, the location of abnormality was identified, and the result is shown in Fig. 14. As shown in Fig. 14, the objective functions $J_{\omega, 1}$ and $J_{2 \omega, 1}$ show the minimum value at the 69th and the 70th element, respectively. Moreover, $J_{\omega, 1}$ shows a significantly small value at the 69th and 70th elements. Therefore, we recognized that an abnormality might occur at the 69th or the 70th element.

We next identified the cause and the severity of the abnormality. From the abnormal response, we assumed that the cause had nonlinear property, and in this study we assumed that a breathing crack was the cause of the abnormality. A breathing crack occurs at a nodal point, so the location of the breathing crack was set at the 70th nodal point, between the 69th and 70th elements. The abnormal response under the breathing crack was calculated by using the FEM based on the mixed variational principle ${ }^{(25)}$.

The objective functions $J_{\omega, 2}$ and $J_{2 \omega, 2}$ were calculated for various crack depths, and the results are shown in Fig. 15. The objective functions are normalized by the minimum value of each objective function. From the figure, the depth is determined to be $34 \%$ from $J_{\omega, 2}$ or $42 \%$ from $J_{2 \omega, 2}$. The change of the objective function $J_{\omega, 2}$ is larger than that of $J_{2 \omega, 2}$, and thus the final result is determined to be $34 \%$ in this case. In this example, we decided the final result as above because this is a primary diagnosis. For more accurate diagnosis result, a precise diagnosis approach, which is, for example, a nonlinear analysis of the beam with multiple causes, will be needed.

The exact abnormality is a breathing crack of $40 \%$ at the 71 st nodal point, while the identified one is a breathing crack of $34 \%$ at the 70 th nodal point. In our previous studies ${ }^{(20)-(22)}$, the abnormality was an additional mass, and it could be easily realized in the experiment, while the breathing crack was difficult to realize. We consider that the diagnosis result was slightly incorrect with the exact one because the response change was not caused only by a breathing crack.

Moreover, in this experiment, the breathing crack was located at the nodal point while a crack will be located not only at the nodal point. At that time, the element which includes the crack location will be estimated as the location of abnormality, and then, the crack is assumed at each end of the element and the severity of the crack will be identified.

\section{Conclusion}

In this study, a stepwise diagnostic approach for a beam with an abnormality of 
nonlinear property, that is, a breathing crack, was proposed. In that method, the location and the severity of abnormality were identified by using the multi-frequencies of response under the abnormal condition. Using the numerical example of a uniform beam fixed at both ends, the validity of the method was confirmed, and we determined that the selection of the excitation frequency was very important. Moreover, the applicability of the method was confirmed by using experimental data. Therefore, we conclude that the proposed diagnosis method is feasible for actual applications with breathing crack abnormalities.

\section{Acknowledgments}

The authors would like to thank Prof. Keisuke Kamiya (Aichi Institute of Technology) for valuable discussions about nonlinear simulations. This work was supported in part by research grants from JSPS Grant-in-Aid for Scientific Research (C) No. 21560238.

\section{References}

(1) Hiroshi Mo, Shin-ichi Yamada, Hideji Fujikawa, Improvement of Reasoning Model for Fuzzy Diagnosis System, Japan Society for Fuzzy Theory and Systems, Vol.5, No.3, (1993), 565-576.

(2) Shinji Utsumi, Zen-ichiro Kawasaki, Kenji Matsu-ura, Masatake Kawada, Discrimination of Wear Particle in Lubricating Oil to Diagnosis the Bearing using Wavelet Transform and Fuzzy Systems Theory, Transactions of the Institute of Electrical Engineers of Japan.B, Vol.119, No.2 (1999), 147-155.

(3) Yasuyuki Oguma, Masahiro Tsunoyama, Youichi Jinnno, Masayuki Ogawa, Tatsuo Sato, Development and Evaluation of a System for Facility Diagnosis of Rotating Machines, The Institute of Electronics, Information and Communication Engineers Technical Report DC2005-78, (2006), 37-42.

(4) $\mathrm{Ke} \mathrm{Li}$, Peng Chen, Huaqing Wang, Hongtao Xue, Condition diagnosis for rotating machinery using dimensional \& non-dimensional symptom parameters, Journal of the Society of Plant Engineers Japan, Vol.21, No.3, (2009), 115-122.

(5) $\mathrm{Ke} \mathrm{Li}$, Peng Chen, Huaqing Wang, Hongtao Xue, Sequential diagnosis method for rotating machinery using fuzzy neural network \& symptom parameters in frequency domain: Application on the condition diagnosis of structural fault of rotating machinery, Journal of the Society of Plant Engineers Japan, Vol.22, No.2, (2010), 62-70.

(6) Maosen Cao, Lin Ye, Limin Zhou, Zhongqing Su, Runbo Bai, Sensitivity of fundamental mode shape and static deflection for damage identification in cantilever beams, Mechanical Systems and Signal Processing, Vol.25, No.2,(2011), pp.630-643.

(7) Young Cheol Huh, Tae Young Chung, Seok Jun Moon, Hyun Gwon Kil, Jae Kwan Kim, Damage detection in beams using vibratory power estimated from the measured accelerations, Journal of Sound and Vibration, Vol.330, No.15 (2011), pp.3645-3665.

(8) Ugo Andreaus, Paolo Baragatti, Cracked beam identification by numerically analysing the nonlinear behaviour of the harmonically forced response, Journal of Sound and Vibration, Vol.330, No.4 (2011), pp. 721-742.

(9) Animesh Chatterjee, Structural damage assessment in a cantilever beam with a breathing crack using higher order frequency response functions, Journal of Sound and Vibration, Vol.329, No.16 (2010), pp.3325-3334.

(10) Jinhee Lee, Identification of multiple cracks in a beam using vibration amplitudes, Journal of Sound and Vibration, Vol.326, No.1-2 (2009), pp.205-212.

(11) Jinhee Lee, Identification of multiple cracks in a beam using natural frequencies, Journal of Sound and Vibration, Vol.320, No.3 (2009), pp.482-490.

(12) Ricardo Perera, Sheng-En Fang, C. Huerta, Structural crack detection without updated baseline model by single and multiobjective optimization, Mechanical Systems and Signal Processing, Vol.23, No.3 (2009), pp.752-768. 
(13) Z.K. Peng, Z.Q. Lang, S.A. Billings, Crack detection using nonlinear output frequency response functions, Journal of Sound and Vibration, Vol.301, No.3-5 (2007) 777-788.

(14) L.Z. Guo, S.A. Billings, Detection of fatigue cracks in a beam using a spatio-temporal dynamical system identification method, Journal of Sound and Vibration, Vol.299, No.1-2 (2007) 22-35.

(15) G.Y.Xu, W.D.Zhu, B.H.Emory, Experimental and Numerical Investigation of Structural Damage Detection Using Changes in Natural Frequencies, Transaction of the ASME, Journal of Vibration and Acoustics, Vol.129, No.6(2007), pp.686-700.

(16) S. Sekhar, Crack identification in a rotor system: a model-based approach, Journal of Sound and Vibration, Vol.270, No.4-5 (2004), pp.887-902.

(17) N.Bachschmid, P.Pennacchi, A.Vania, Identification of multiple faults in rotor system, Journal of Sound and Vibration, Vol.254, No.2(2002), pp.327-366.

(18) Paolo Pennacchi, Nicolò Bachschmid, Andrea Vania, Gian Antonio Zanetta, Luca Gregori, Use of modal representation for the supporting structure in model-based fault identification of large rotating machinery: part 1 - theoretical remarks, Mechanical Systems and Signal Processing, Vol.20, No.3 (2006), pp.662-681.

(19) Paolo Pennacchi, Robust estimate of excitations in mechanical systems using M-estimators - Theoretical background and numerical applications, Journal of Sound and Vibration, Vol.310, No.4-5 (2008), pp.923-946.

(20) Shozo Kawamura, Hiroaki Tao, Hirofumi Minamoto, Hossain Md. Zahid, Development of a stepwise primary diagnosis method of a beam using a force identification and optimization approach, Journal of Sound and Vibration, Vol.311, No.3-5 (2008), pp.861-873.

(21) S.Kawamura, J.Yamamoto, H.Minamoto, Model-based diagnosis approach for a beam structure using the modified mathematical model, Journal of System Design and Dynamics, Vol.5, No.2 (2011), pp.306-319.

(22) S.Kawamura, J.Yamamoto, H.Minamoto, A regularization method for a stepwise primary diagnosis method of a beam structure using a force identification technique, Journal of System Design and Dynamics, Vol.5, No.4 (2011), pp.612-623.

(23) S.Kawamura, K.Niimi, H.Minamoto, Proposition of a diagnosis method of rotating machinery using a force identification approach, CD-ROM Proceedings of the 17-th International Congress on Sound and Vibration, No.570, (2010).

(24) T.G.Chondros, A.D.Dimarogonas, J.Yao, Vibration of a beam with a breathing crack, Journal of Sound and Vibration, Vol.239, No.1 (2001), pp.57-67.

(25) K.Kamiya and T.Yoshinaga, Nonlinear Steady-State Vibration Analysis of a Beam with Breathing Cracks, Journal of System Design and Dynamics, Vol.2, No.3 (2008), pp.750-761. 